\title{
Quality, cost and outcomes measures in orthopaedic healthcare
}

\author{
Cyril Mauffrey $^{1} \cdot$ Andreas F. Mavrogenis $^{2}$
}

Received: 25 July 2017 / Accepted: 2 August 2017 / Published online: 9 August 2017

(C) SICOT aisbl 2017

In the era of patient-centred healthcare, good quality medical practice represents an intervention resulting in outcomes that match patients' expectations. However, good quality healthcare is not without a cost, and outcomes measurement is complex and flawed by the multiple layers that exist and define such measurements. To meet the financial challenges faced in the healthcare industry, healthcare organizations are striving to control operational costs. Costing is essential to maximize the resources available to the healthcare providers and increase the services offered to patients. Still, patients' expectations in healthcare continue to increase; patients' expectations are diverse, and subject to variability in space (culture, religion, education, social status, profession, secondary gains) and time. Understanding and adequate management of patients' expectations can enhance their satisfaction level, improve outcomes and decrease liability. In this setting, assessment of outcomes has become increasingly more important in the healthcare industry. This editorial paper discusses the quality and cost of healthcare, the expectations and variances of patients, and the measurements of outcomes for patient-centred care into existing health care systems (Figs. 1 and 2).

Andreas F. Mavrogenis

afm@otenet.gr

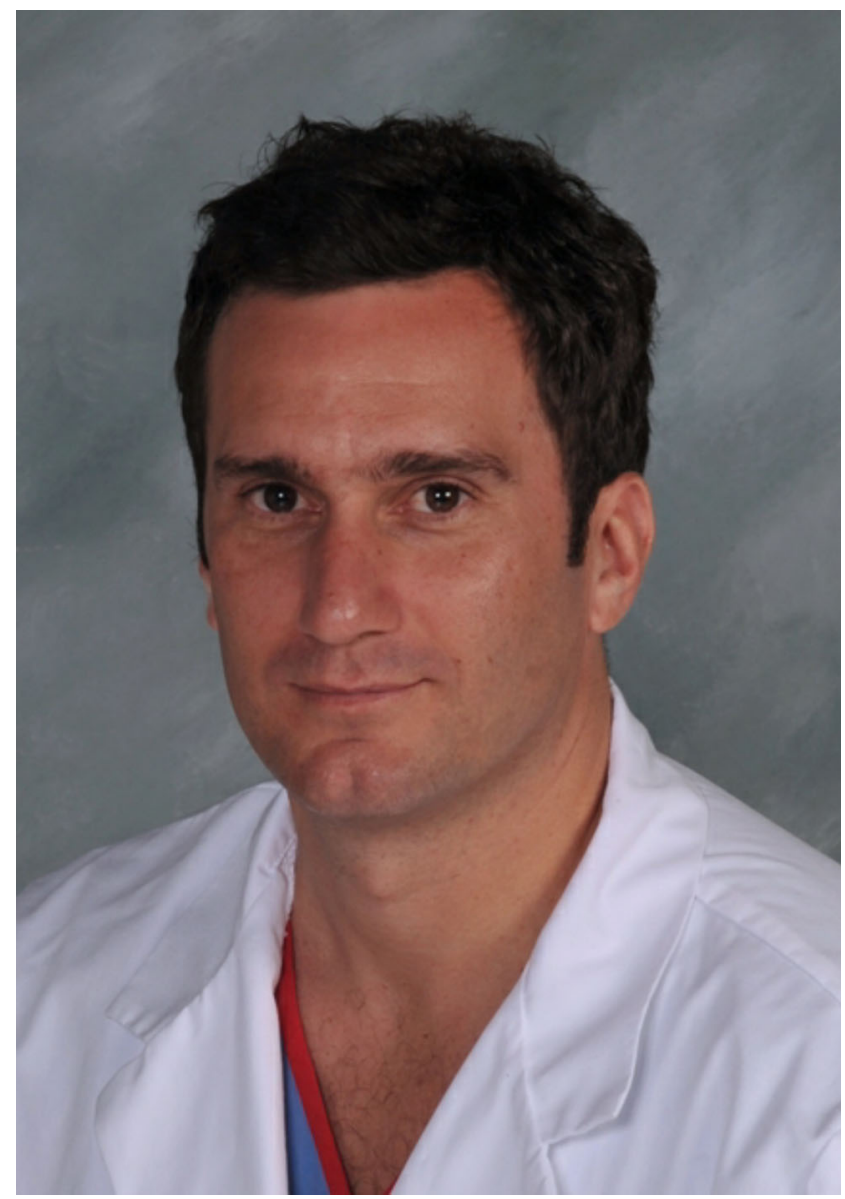

Fig. 1 Cyril Mauffrey

\section{Quality and cost of healthcare}

Department of Orthopaedics, Denver Health Medical Center, Denver, USA

2 First Department of Orthopaedics, National and Kapodistrian University of Athens, School of Medicine, 41 Ventouri Street, 15562 Cholargos, Athens, Greece

Debate over healthcare often focuses on two key issues: quality and cost. Quality problems may not be the result of 


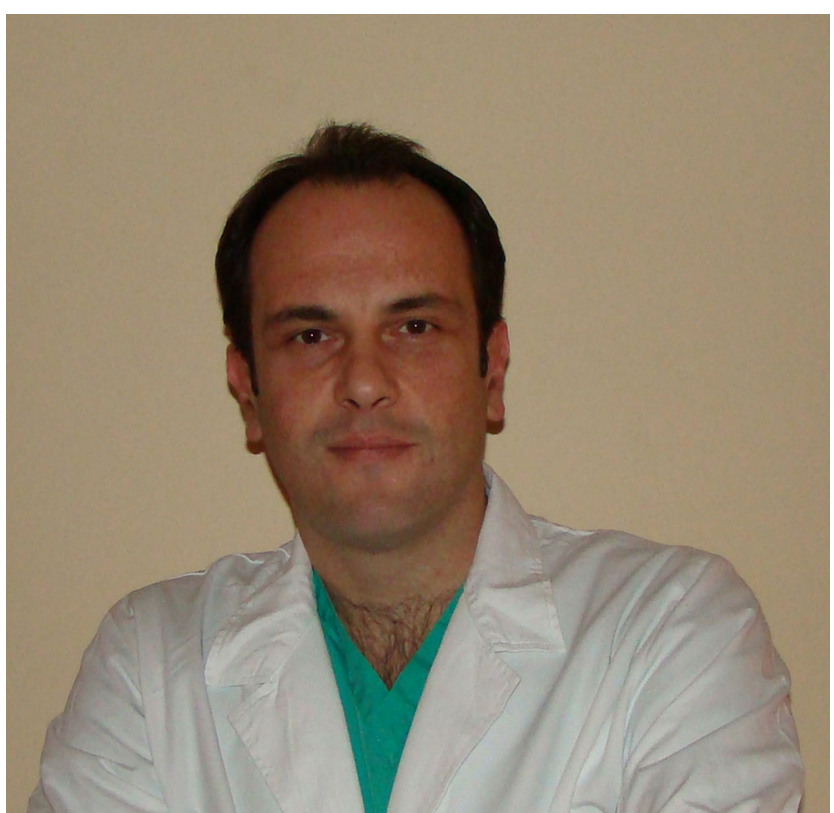

Fig. 2 Andreas F. Mavrogenis

financing constraints but rather a significant contributor to funding pressures. Ultimately, focus on quality would provide an effective strategy to contain costs, not to mention having a positive impact on patient well-being. Why is healthcare spending so high? And do healthcare systems really deliver less quality for the amount of money spent by other industrialized nations? U.S. healthcare spending was rising at 3.6\% between 1996 and 2006, lower than the very high levels of $4.3 \%$ and $4.8 \%$ in the UK and New Zealand, respectively. Where is all this money going? Wealthier countries pay more in their economy for healthcare, population is getting older and sicker, population is using more healthcare, healthcare is using more technology, and higher prices are charged for the same goods and services. Among key healthcare risk factors are smoking, alcohol use and obesity that translate into a high number of patients with chronic cardiovascular disease and diabetes. These seem to explain for the most part the overall heavier healthcare expenditures [1].

Technologies for basic medical care, diagnostics, hospitals, pharmaceuticals, and nursing homes are similar in healthcare systems in U.S. and Europe. However, this is not the fact for the costs for the same care; drugs, hospital and outpatient visits, diagnostic and therapeutic procedures cost a lot different in similar healthcare systems [1]. Does this spending correlate into better outcomes? Outcome measures could clearly answer this question. Morbidity and mortality rates in U.S. and European hospitals are just average. Therefore, prevention instead of management a disease is necessary. Social welfare spending, income inequality and accessibility of high-risk consumer products such as tobacco and alcohol use, and poor food quality have a tremendous impact on disease rates beyond the control of the healthcare system [1].
Quality costing in healthcare organizations may present solutions to reduce waste and improve performance. Additionally, improving quality should aim to reduce the cost of services. Example is the cost of failures to healthcare; these costs can be significant if one considers hospital revisits, waiting lists, medications and re-operations. Non-technical aspects of quality such as attitude of staff and their behaviour, which may be more difficult to measure, and cost are more influential in patients' rating of quality than the technical aspects $[2,3]$. Often, quality costs only focus on the cost to the organization in an effort for profit making. In this setting, healthcare quality may be underestimated if the cost to the patients is not taken into account. Examples of patients' cost include travelling costs (revisiting the hospital), time consumption (waiting areas), travelling costs for a second opinion and expenses for repeating investigations abroad due to poor counselling [3].

When intangible costs of quality are taken into account, the agreed expectations of patients which are excessively identified in order to provide reasonable quality of care are difficult to determine and measure. To the best of our knowledge, there has not been much progress in establishing standard measures of program activities as activity based costs for health services. Information on the number of patients seen and the quality of services provided in a patient-oriented approach is often not available [3]. The cost of quality is measured by the cost paid by the hospital or part of it, but it does not take into account the cost of training and experience or stress to staff, and the cost to the society, families, and other agencies such as court, foster care, and police. Such costs may be more difficult to estimate due to the variability of cases and complexity of such processes [3]. Therefore, defining quality is challenging, and measuring patients' outcomes, expectations and satisfaction is important.

\section{Cultural and societal variances in patients' expectations}

Studying, training and practicing medicine on different continents, a surgeon may see first hand how patients' expectations may differ based on their specific needs and beliefs; kneeling to pray in the Middle East or beyond, strong desire to have all hardware removed in China, reluctance in accepting below knee amputation in Central and parts of Latin America, narcotic use epidemics in North America, are among many factors that may affect patients' expectations. Measurement of outcomes may be affected by the time waited to see the surgeon, the crowded waiting room and 'life events' such as blowing up a tire on the way to the follow up appointment or other mishaps.

It is striking to see in practice how pain is subjective and how the 'post-operative experience' varies geographically. The rare 'opiate virgins' as they are called in the US need significantly more pain medication following a minor soft tissue procedure 
than post polio syndrome patients that I have operated on in India who were happy and thankful with a pair of Paracetamol. If the quality of my surgery was rated by the minimal use of analgesia post-operatively and/or patients' satisfaction, I was a better surgeon then that I am now. How could this subjective experience not affect functional outcome scores?

\section{Outcomes assessment in healthcare}

Measuring outcomes and quality of health care is a valid process as long as the provision of care is standardized. This later mission is arduous within one institution let alone in a state or region. For low and middle-income countries (LMIC) the challenge is immense. The focus for those countries (and ours) is to reduce the burden of post surgical infection. The holy-grail remains prevention, which goes hand in hand with education.

World Health Organization (WHO) has led the way in terms of data collection, surveillance and outcomes in LMIC especially for surgical site infection (SSI) prevention. The recently published WHO recommendations on pre-, intraand post-operative methods for surgical site infection prevention represent a superb effort to serve such a population $[4,5]$. The aim is to highlight methods that may help reduce the two to 20 times higher prevalence of health-care associated infections of which surgical site infections are the most common in LMIC $[6,7]$. These guidelines also serve as recommendations for high-income countries (HIC) where SSI are proportionally less frequent yet representing one of the largest burden in terms of cost to patients, hospitals, payers and society in general. Systematic review and metanalyses $[6,7]$ quoting such prevalence of SSI in LMIC are without doubt an underestimation of the genuine infection rates. Published recommendations are an outstanding document that deserves to be placed in every clinic and operating rooms for the benefit of the patients and their expectations. The set of 13 pre-operative and 16 intra and post-operative recommendations take into account a global cost-effective perspective. The challenge remains the dissemination of the document, its application and more importantly implementation of quality surveillance measures to quantify and qualify trends of health-care related infections in areas that need it the most.

\section{Value-based healthcare today}

Objectively measuring outcomes in LMIC is undoubtedly a challenge because of cost issues, language barriers between different ethnicities, distance to the point of healthcare, and losses to follow-up. Indigent population in High-income countries such as in the USA or Europe may represent a similarly arduous task for identical reasons. The recent trend towards value-based healthcare emphasizes the importance of continuous outcome assessment, process improvement strategies and rigorous surveillance of post-surgical complications; unfortunately, these processes have swallowed a large slice of the working budget of departments and institutions.

Denver Health Medical Center is a nationally renowned model of integrated healthcare system with a structured approach to quality and safety. More than half of the hospital discharges are either uninsured or Medicaid patients. These patients with difficult access to healthcare include the poorest, non-English speakers, substance abusers, homeless, chronically severely mentally ill, and ethnic minorities [8]. In the last several years, Denver Health provided several hundreds of millions of dollars of uncompensated care to patients with no insurance. Its operating margin in 2012 was only $0.4 \%$ leaving few resources for quality and safety initiatives [8]. Despite tight financial budgets, the implementation of a lean approach, a vertically fully integrated system, a passionate and dedicated workforce employed by the Hospital have helped Denver Health ranked highly among 121 leading academic hospitals in the US (University HealthSystem Consortium) [8]. However, and despite such efforts, routine implementation of functional outcome measurement in such urban/county setting remains a challenge. Patients do not always reliably follow-up, and getting hold of patients through the phone or by mail may be difficult for a large proportion of our homeless population. In addition, the implementation of Patient Reported Outcome Measures (PROMS) using modern computerized adaptive test formats (such as the PROMIS score [9]) which allows the test to customize questions based on patients response to the previous item, is problematic due to cost of the IT support required for routine implementation. In today's Europe hospitals admitting low-income patients, the situation is similar. Orthopaedic surgery has evolved, however, the susceptible population such as the elderly, immuno-suppressed, intravenous drug users, third-country migrants, prisoners have increased significantly. This phenomenon has raised costs of health care, reduced funding, shortened follow-up, precluded outcome measurements, and neglected patients' expectations.

\section{Orthopaedic trauma research in healthcare}

How do we ensure that what we do for our patients is a procedure that they will benefit from? How do we know that with the increasing social, ethnical, and cultural disparities the tools that we use to assess quality of our intervention are sufficiently accurate? Most likely we don't.... and we must as a profession drive the process, with our patients, of defining what is quality and what is not, what value is and what is not by understanding more accurately our patients need and expectations.

Incorporating research in the setting of healthcare can be rewarding. However, how does research apply to healthcare? Research starts with the patients and ends to the patients. 
Surgeons may help thousands of patients with their knowledge, transfer of experience, education and research. The goal is to improve the quality of healthcare, thereby to improve the quality, cost and outcomes of the patients. Research starts with accurate data, statistical analysis and presentation of the results, and scientific writing. By studying a process, improvements can be applied to the most appropriate aspect of the process and outcomes can be evaluated and measured. For example, the outcomes of the patients with calcaneal fractures can be ensured [10], the risk of bleeding of hip fracture patients taking clopidrogel can be estimated [11], the use of antiosteoporotic medication in elderly patients with fragility pelvic fractures can be classified [12], and traditional treatments in trauma can be revisited [13-18].

This was the purpose of this special issue; it was an attempt to create a summary that the readers would find interesting and didactive. The authors provided scholastic original and review papers with current indications, techniques, limitations, pros and cons, complications, outcomes and related metrics in the context of the ever-growing, brave field of orthopaedic trauma. Although quality costing was not a fundamental purpose, we believe that this concept will expand in the next years allowing healthcare providers to achieve improved healthcare for the patients' outcome.

\section{Funding None.}

\section{Compliance with ethical standards}

Conflict of interest All authors declare that they have no conflict of interest.

No benefits have been or will be received from a commercial party related directed or indirectly to the subject matter of this article.

Ethical approval All procedures performed in studies involving human participants were in accordance with the ethical standards of the institutional and/or national research committee and with the 1964 Helsinki declaration and its later amendments or comparable ethical standards.

Informed consent Informed consent was obtained from all individual participants included in the study.

\section{References}

1. https://epianalysis.wordpress.com/2012/07/18/usversuseurope. Accessed on July 10, 2017

2. Soliman AA (1992) Assessing the quality of health care: a consumerist approach. Health Mark Q 10(1-2):121-141

3. Al Rashdi I (2011) How much the quality of healthcare costs? A challenging question! Oman Med J 26(5):301-302

4. Allegranzi B, Bischoff P, de Jonge S, Kubilay NZ, Zayed B, Gomes SM, Abbas M, Atema JJ, Gans S, van Rijen M, Boermeester MA, Egger M, Kluytmans J, Pittet D, Solomkin JS, WHO Guidelines
Development Group (2016) New WHO recommendations on preoperative measures for surgical site infection prevention: an evidencebased global perspective. Lancet Infect Dis 16(12):e276-e287

5. Allegranzi B, Zayed B, Bischoff P, Kubilay NZ, de Jonge S, de Vries F, Gomes SM, Gans S, Wallert ED, Wu X, Abbas M, Boermeester MA, Dellinger EP, Egger M, Gastmeier P, Guirao X, Ren J, Pittet D, Solomkin JS, WHO Guidelines Development Group (2016) New WHO recommendations on intraoperative and postoperative measures for surgical site infection prevention: an evidence-based global perspective. Lancet Infect Dis 16(12):e288-e303

6. Allegranzi B, Bagheri Nejad S, Combescure C, Graafmans W, Attar H, Donaldson L, Pittet D (2011) Burden of endemic health-careassociated infection in developing countries: systematic review and meta-analysis. Lancet 377(9761):228-241

7. Bagheri Nejad S, Allegranzi B, Syed SB, Ellis B, Pittet D (2011) Health-care associated infection in Africa: a systematic review. Bull World Health Organ 89:757-765

8. Mehler PS (2014) Public safety-net hospitals - the Denver health model. In: patient safety in surgery. Stahel PF, Mauffrey C (Eds). Springer-Verlag London 245-264

9. Brodke DJ, Saltzman CL, Brodke DS (2004) PROMIS for Orthopaedic outcomes measurement. J Am Acad Orthop Surg 24:744-749

10. Qiang M, Chen Y, Jia X, Zhang K, Li H, Jiang Y, Zhang Y (2017) Post-operative radiological predictors of satisfying outcomes occurring after intra-articular calcaneal fractures: a three dimensional CT quantitative evaluation. Int Orthop Jul 27. doi: 10.1007/s00264017-3577-0. [Epub ahead of print]

11. Pailleret C, Ait Hamou Z, Rosencher N, Samama CM, Eyraud V, Chilot F, Baillard C (2017) A retrospective comparison between delayed and early hip fracture surgery in patients taking clopidogrel: same total bleeding but different timing of blood transfusion. Int Orthop Jul 25. doi: 10.1007/s00264-017-3571-6. [Epub ahead of print]

12. Kanakaris NK, Greven T, West RM, Van Vugt AB, Giannoudis PV (2017) Implementation of a standardized protocol to manage elderly patients with low energy pelvic fractures: can service improvement be expected? Int Orthop Jul 21. doi: 10.1007/s00264-0173567-2. [Epub ahead of print]

13. Nowak LL, Vicente MR, McKee MD, Hall JA, Nauth A, Schemitsch EH (2017) Orthopaedic surgeons' opinions surrounding the management of proximal humerus fractures: an international survey. Int Orthop Jul 20. doi: 10.1007/s00264-017-3569-0. [Epub ahead of print]

14. Papin P, Berthonnaud E Incidence of osteosynthesis of members in France. Int Orthop 41(8):1501-1506

15. Seng P, Traore M, Lavigne JP, Maulin L, Lagier JC, Thiery JF, Levy PY, Roger PM, Bonnet E, Sotto A, Stein A (2017) Staphylococcus lugdunensis: a neglected pathogen of infections involving fracturefixation devices. Int Orthop 41(6): 1085-1091

16. Thés A, Klouche S, de Tienda M, Bauer T, Hardy P (2017) Cortical onlay strut allograft with cerclage wiring of periprosthetic fractures of the humerus without stem loosening: technique and preliminary results. Eur J Orthop Surg Traumatol 27(4):553-557

17. Valenti P, Aliani D, Maroun C, Werthel JD, Elkolti K (2017) Shoulder hemiarthroplasty for proximal humeral fractures: analysis of clinical and radiographic outcomes at midterm follow-up: a series of 51 patients. Eur J Orthop Surg Traumatol 27(3):309-315

18. Maroun C, Aliani D, Hass A, Werthel JD, Vidil A, Valenti P (2017) Shoulder arthroscopy combined to hardware removal in proximal humeral fractures: a series of 58 cases with a mean follow-up of 2 years. Eur J Orthop Surg Traumatol 27(3):317-321 